\title{
An Ultrastructural Study of the Effects of Aeration and Physical Activity on Aging in Tetrahymena pyriformis '
}

\author{
ALFRED M. ELLIOTT, DAVID M. TRAVIS AND JANIS A. WORK \\ Department of Zoology, The University of Michigan, Ann Arbor, and \\ Departments of Pharmacology, Therapeutics, and Medicine, \\ University of Florida, Gainesville
}

\begin{abstract}
The ultrastructure of $T$. pyriformis, strain $\mathbf{E}$, is markedly influenced by the physical conditions of the medium during the growth cycle. Logarithmic growth cells contain a minimal number of mitochondria which are peripherally distributed. Rough endoplasmic reticulum ( $R E R$ ) is abundant and numerous primary lysosomes are scattered throughout the cytoplasm. Lipid droplets are sparse. This situation is unchanged in quiet culture flasks or those that are aerated and/or stirred. When cells enter stationary growth the physical treatment of the medium has profound effects on the cytoarchitecture. In quiet flasks the mitochondria have increased several times over that in young cultures and they are randomly distributed. Lipid droplets are abundant. Very little RER can be seen and primary lysosomes are scarce. A few autophagic vacuoles appear at this phase of growth. When the culture medium has been stirred throughout the growth cycle the ciliates appear quite different. No lipid droplets are formed; the mitochondria are few and peripherally distributed as in young cells. Primary lysosomes and RER are scarce. An occasional autophagic vacuole is observed. Aeration of the medium does not alter this cytoarchitecture. Physical activity of the cells seems to influence the distribution, number, and kinds of particulates. These observations suggest that certain metabolic processes as reflected by ultrastructure are dependent on the physical activity of the ciliates during the growth cycle.
\end{abstract}

In an earlier report it was demonstrated that Tetrahymena pyriformis undergoes cytological changes correlated with cultural aging of the cells (Elliott and Bak, '64b). These involve the structure, number, and distribution of mitochondria, the accumulation of lipid, and the occurrence of autophagic vacuoles (cytolysomes). This sequence of events was observed in cells grown in flasks without agitation or aeration. In a more recent study aeration and physical agitation were introduced to promote gas exchange. Under these conditions nearly all of the cytological changes noted in aging cells grown in undisturbed flasks failed to appear. The purpose of this communication is to describe the ultrastructural changes that occur during the growth cycle when the cells are grown under various culture conditions.

\section{MATERIALS AND METHODS}

$\mathrm{T}$. pyriformis, strain $\mathrm{E}$, was cultured axenically in $1,000 \mathrm{ml}$ Erlenmeyer flasks containing $200 \mathrm{ml}$ of medium. The flasks were stoppered with plastic plugs (Scientific Products, Evanston, Illinois) which were pierced with $5 \mathrm{~mm}$ diameter glass tubing pulled out to a capillary of $1 \mathrm{~mm}$ diameter at the end extending into the flask. The tip of the capillary was placed $0.5 \mathrm{~cm}$ from the surface of the medium and the opposite end connected to a suction line. When desired, a gentle flow of air was drawn through the flask. The rate was such as not to agitate the medium. The flasks were incubated at $26^{\circ} \mathrm{C}$ in a plastic water bath. Teflon-coated magnets (Cole-Palmer, Chicago, Illinois) were placed in the flasks and sterilized with the medium. Preliminary tests demonstrated that Teflon had no adverse effects on the ciliates. The magnetic stirrers were placed under the flasks outside the bath and when desired they were set in motion at approximately 150 revolutions per minute.

1 This investigation was supported by a research grant AI 01416-13 from the National Institute
Allergy and Infectious Diseases, N.I.H., U.S.P.H.S. 
The medium consisted of $2 \%$ proteosepeptone (Difco Laboratories, Detroit, Michigan), $0.5 \%$ glucose, $0.005 \%$ liver extract and glass distilled water. The $\mathrm{pH}$ was set at 7.2. The initial innoculation consisted of logarithmic growth cells in such numbers as to provide approximately 2,000 cells per $\mathrm{ml}$ of culture medium in the flasks. The culture flasks were set up so that the following conditions were met: (1) Quiet (unstirred and unaerated); (2) Quiet but aerated; (3) Agitated (stirred) but not aerated; (4) Agitated and aerated. At specified intervals samples were withdrawn from the flasks, centrifuged into soft pellets, and fixed for 20 minutes in either cold glutaraldehyde ( $3 \%$ buffered with cacodylate at $\mathrm{pH} 7.2$ ) and post-fixed in $1 \% \quad \mathrm{OsO}_{4}$, or in $1 \% \mathrm{OsO}_{4}$ buffered with $0.14 \mathrm{M}$ Veronal acetate $(\mathrm{pH} 7.4)$ containing $45 \mathrm{mg}$ of sucrose per $\mathrm{ml}$. The ciliates were then dehydrated with graded ethanols and embedded in Epon 812 according to the method of Luft ('61). The blocks were polymerized for three days at graded temperatures $\left(37^{\circ}-60^{\circ} \mathrm{C}\right)$. The embedded pellets were sectioned with a Porter-Blum MT-2 microtome and examined in an RCA EMU 3E. All experiments were repeated several times and the most typical reported here.

\section{RESULTS}

Earlier studies have shown that this strain (E) of $T$. pyriformis goes through the growth cycle in about six days of incubation when grown at $25-26^{\circ} \mathrm{C}$ in $2 \%$ proteose-peptone (supplemented with glucose) without aeration or agitation (Elliott and Hogg, '52). Exponential growth, which lasts about 48 hours, is followed by a gradual decrease in cell division rate until the maximal stationary growth phase is reached in about six days. The present study is concerned with cells in exponential and stationary growth, hence samples for study were taken at 36 hours and seven days of incubation. Checks on cell numbers assured us that even though aeration and stirring were introduced the cells were always in exponential growth at 36 hours and stationary growth at seven days.

\section{Exponential growth}

When examined with the phase contrast microscope ciliates in logarithmic growth possess essentially the same characteristics in all four flasks. They are elongated and are constantly swimming at a rapid rate. Their cytoplasm appears translucent; a few lipid droplets can be observed in the anterior region of the cell. A small percentage of the cells are in various stages of division.

At the ultrastructural level the internal morphology is also similar under the four culture conditions. The mitochondria are usually oval in shape and are concentrated near the plasma membrane (fig. 1). An occasional mitochondrion contains a dense granule (intramitochondrial mass). Rough endoplasmic reticulum (RER) is abundant and randomly distributed throughout the cytoplasm. Sometimes it is arranged in lamellae which are often associated with mitochondria (fig. 2). A few lipid droplets appear in the sections. Primary lysosomes (de Duve, '64) are numerous. Autophagic vacuoles are never seen. The nucleoli are located near the macronuclear membrane and are typically crescent in shape (figs. 1 and 8 ).

\section{Stationary growth}

Whereas no behavioral or morphological differences can be detected in exponentially growing ciliates under the four culture conditions, marked differences appear when the cells reach the stationary phase of growth. These variations in morphology and activity are directly correlated with the conditions of the environment. Each is considered separately.

1. Quiet flask. After seven days of culture in the undisturbed and unaerated flask, the ciliates are sluggish and have a pronounced pyriform shape. Their cytoplasm is dense owning to the great numbers of mitochondria and lipid droplets. These morphological details can be observed more critically at the ultrastructural level.

The mitochondria are uniformly spherical in shape, are randomly distributed throughout the cytoplasm, and are more numerous than in younger cultures (fig. 3). The mitochondria contain a dense granule in about the same frequency as in logarithmic growth cells. The numerous lipid droplets are concentrated anterior to the macronucleus although they can be 
seen throughout the cytoplasm. The cytoplasm is packed with free ribosomes but RER is sparse. Only an occasional primary lysosome can be observed in the sections. A few autophagic vacuoles (fig. 7) appear at this stage but are not seen in all sections. In most cells the nucleoli are few in number, large, and often assume irregular shapes (figs. 3 and 9). They may result from the fusion of the smaller crescent-shaped nucleoli typical of younger cells as described by Cameron and Guile ('65).

2. Aerated flask. After seven days of aeration with forced air but no stirring, the cells resemble those in the preceding experiment as regards activity and shape. However, they appear somewhat more dense, indicating a higher concentration of cytoplasmic particulates.

Electron micrographs of these cells reveal that the morphology and distribution of the mitochondria are essentially the same as in the preceding experiment (fig. 4). However, the number is greater and many more contain a dense granule. RER occurs in modest amounts (fig, 5). Primary lysosomes are sparse, indeed they are rarely seen in sections. A few autophagic vacuoles are present although none can be clearly seen in figure 4. Lipid droplets are abundant and are concentrated anterior to the nucleus. They often take on a stellate shape (fig. 5). The nucleoli may or may not resemble those of younger cells of the same age. This characteristic seems to vary from cell to cell.

3. Agitated flask. When the flask is stirred continuously for the seven days of incubation but not aerated, the cells are strikingly different when compared with ciliates that have not been agitated. Their general appearance is that of exponentially growing cells. They are active and their cytoplasm contains little granular material. Electron micrographs confirm the light microscope observations.

The mitochondria are few in number and are peripherally located (fig. 6). Very few contain dense granules. Most of the ribosomes are free in the cytoplasm although some RER is observed enveloping food vacuoles. Primary lysosomes are present in modest numbers and autophagic vacuoles containing a single mitochondrion are numerous, suggesting that excess mitochondria are being disposed of by hydrolysis. Lipid droplets are scarce which suggests that the cells are able to store very little energy in this form when being constantly agitated. This is in sharp contrast to ciliates that grow in quiet flasks where enormous amounts of lipid are stored. The nucleoli are usually fused into large bodies and may be centrally located as shown in figure 6 . The fate of the nucleolar material (ribosomes) seems to resemble that in some cells of the same age but not stirred or aerated. (Compare figures 3 and 6 .)

4. Agitated and aerated flask. When observed under the phase contrast microscope, stationary phase cells that are continuously stirred and aerated have the same behavior and morphology as those in the preceding experiment. The addition of aeration seemed to have little or no effect on the ciliates.

At the ultrastructural level the ciliates also show essentially the same structure as those that are not aerated. For that reason no micrographs of these cells is included in this report. Obviously, continued activity induced by stirring is the critical factor in bringing about the observed morphology rather than aeration.

When the ciliates are continued in the stirred and aerated flasks for another five days (12 days of incubation) other ultrastructural changes are noted when compared to seven-day-old cells (fig. 10). The mitochondria diminish somewhat in number but retain their peripheral position. The cytoplasm contains very little RER although free ribosomes are abundant. An occasional autophagic vacuole appears in the cytoplasm. Food vacuoles are present which often contain fragments of other ciliates. T. pyriformis is cannibalistic in old cultures where high mortality occurs. Lipid is conspicuously absent. The nucleoli are spherical, small, and few in number, quite unlike young or seven-day-old cells.

\section{DISCUSSION}

The physiological and morphological changes that occur in microorganisms as they go through the growth cycle are regarded as characteristics associated with aging. This type of aging should be distinguished from clonal aging which occurs 
among some ciliates (Sonneborn, '57). Sexual strains of $T$. pyriformis, for example, age over long periods of time in test tube culture. They are said to become senile apparently as a result of the accumulation of deleterious genes through mutations. Unless conjugation interrupts this trend, such strains may eventually die out. One symptom of this type of aging is the loss of the micronucleus. However, some amicronucleate strains have been in culture for many years and still retain their original reproductive capacity. These apparently never age in the sense that sexual strains do, and strain $E$ is in this category. In this investigation we are concerned only with the changes that are associated with aging as it occurs during the growth cycle.

1. Mitochondria. The typical logarithmic growth ciliate shows elongated and dividing mitochondria aligned beneath the plasma membrane when grown in quiet flasks (Elliott and Bak, '64b). Dense granules within the mitochondrion are seldom seen. In older, stationary growth phase cells cultured under the same conditions, the mitochondria become spherical, dense granules may be common, the number of mitochondria increases and they are randomly distributed throughout the cytoplasm. There is little, if any, alteration of the size, shape, and configuration of the tubules. Some mitochondria become encapsulated in autophagic vacuoles along with other cellular particulates, and are hydrolyzed.

It is well known that the culture conditions change as the cells pass through the growth cycle (Hall, '53). Wastes accumulate, the $\mathrm{pH}$ may shift up or down, and nutrients are exhausted. Any one or all of these factors may influence the number and distribution of mitochondria. McCashland and Kronschnabel ('62) have demonstrated that as the cells become more crowded the respiratory rate decreases. Certainly ciliates in stationary growth are crowded but contrary to expectations the number of mitochondria is greater than in logarithmic growth cells. However other factors such as adverse levels of oxygen, carbon dioxide, and ammonia may also influence the number of mitochondria.
The distribution of mitochondria may be correlated with metabolic activity as has been shown in metazoan cells (Palade, '56). In young, active cells the mitochondria lie near the kinetosomes and presumably supply energy for the rapidly beating cilia. As the cells age, movement decreases and some mitochondria migrate into the cytoplasm. This change in position is inhibited in old cells that are stirred. The mitochondria remain near the periphery of the cell, just as in young cells. Apparently, when forced by stirring to remain active, the mitochondria retain the same position as in young cells. In other words, their location is correlated with the physical activity of the ciliate.

The normal increase in numbers of mitochondria, characteristic of aging ciliates, is also inhibited by forced physical activity. The numbers remain essentially the same as in exponentially growing cells. However, the presence of autophagic vacuoles in stirred stationary growth phase cells implies that some excess does occur, as in inactive ciliates and that mitochondria are being hydrolized. In very old cells which have been agitated, most of the mitochondria are gone and the remaining few are spherical and peripheral. Perhaps cellular metabolism and activity are at such a low level that only small numbers of mitochondria are necessary to maintain life. The continual destruction of mitochondria with age may be a mechanism the cell possesses for continued existence without exogenous nutrients or under extremely adverse environmental conditions.

Aside from the change in shape, the mitochondria retain their normal morphology during aging. However, some acquire dense granules (intramitochondrial masses) of various shapes. These are most commonly spherical and centrally located; some are rod-shaped and acentric in position. These may be similar to those described by Peachey ('64) for metazoan cells where they were identified as deposits of cations. As yet we have been unsuccessful in identifying these dense granules in the mitochondria of $T$. pyriformis.

Aeration of the culture medium seems to have little effect on the cytological changes associated with aged cells. Since 
there is no difference between cells grown in undisturbed aerated and unaerated flasks it would seem that gas exchange as it occurs under these culture conditions is of little consequence in the aging process. In another series of experiments (unpublished) high levels of carbon dioxide $(25 \%)$ enhanced the aging process but the effects were inhibited when the cultures were stirred. However, it may be that low levels of oxygen in the medium do induce aging. Levy and Scherbaum ('65) have shown that the oxygen in the medium reaches very low levels in early logarithmic growth. The relationship of oxygen tensions to the aging process is the subject of a future investigation.

2. Endoplasmic reticulum. Young ciliates typically contain large amounts of RER, Smooth endoplasmic reticulum (SER) is largely confined to the nephridial tubules which constitute a part of the contractile vacuole system (Elliott and Bak, '64a). The RER is randomly distributed throughout the cytoplasm and is sometimes seen in lamellae in the vicinity of mitochondria and food vacuoles. We have been unable to correlate this regularly arranged RER with any phase of the growth cycle. Such configuration of the RER may be a result of fixation as pointed out by Herndon ('64) in Purkinje cells of rats. Moreover, Steiner et al. ('64) have demonstrated that many environmental factors can elicit responses in the endoplasmic reticulum.

With increasing age, less and less RER is observed in the cytoplasm. That which remains is often located near mitochondria and in some instances seems to be encapsulating cytoplasmic materials, including mitochondria. We have no unequivocal evidence that this actually occurs. However, there seems to be a correlation between the loss of RER and the increase in autophagic vacuoles. The gradual diminishing of RER suggests that protein synthesis is likewise decreasing as the cell ages. Such cells rarely divide which would support this suggestion.

3. Lipid, lysosomes, and autophagic vacuoles. Young ciliates contain numerous primary lysosomes, very few lipid droplets, and no autophagic vacuoles, regardless of which of the four culture con- ditions the cells are exposed to. With increasing age in undisturbed flasks the primary lysosomes decrease in number while lipid droplets and autophagic vacuoles become prominent. When the cells are agitated throughout the growth cycle lipid droplets do not form and autophagic vacuoles are much less numerous.

Lipid accumulation is commonly associated with cellular aging. Lipid-like pigment granules are found in overfed and old Tokophyra and it is suggested that the accumulation of these insoluble particles may be a factor in cell death (Rudzinska, '62). Irwin and Block ('63) have shown that triglycerides increase with increasing age in an unidentified strain (mating type II, variety 1) of T. pyriformis. Presumably these cultures were grown in undisturbed culture media. In the present study with strain E, lipid droplets increase with age also under undisturbed culture conditions but lipid does not accumulate when the cells are forced to move continually. This fact suggests that lipid storage in the form of lipid droplets is accomplished only when the cell is expending little energy for physical activity.

4. Nucleoli. The small peripherally located nucleoli, typical of logarithmic growth cells, undergo fusion as the ciliates enter the stationary phase of growth in strain HSM according to Cameron and Guile ('65). As the cells enter the stationary phase of growth the nucleoli begin to aggregate and eventually fuse forming fewer but larger masses of nucleolar material. This process is reversed when these cells are placed in fresh medium. Protein and RNA synthesis occurs only when the nucleoli are in the unfused condition. The enlarged, irregularly shaped masses of nucleolar material have also been observed in strain $\mathrm{E}$ when the cells are in stationary phase of growth. It is likely that the same process occurs in this strain as in strain HSM, although we have made no effort to confirm the fusion of the small peripherally located nucleoli into the larger bodies.

These studies suggest that physical activity has a profound effect on the metabolism of $T$. pyriformis as reflected by ultrastructural changes that occur during the growth cycle. Biochemical events may be markedly altered by changing the 
physical conditions of the culture medium. This study does not delineate quantitatively what factors are involved when the culture medium is stirred, aerated, or undisturbed. It does, however, point up the fact that such manipulation of the culture medium does indeed induce striking changes in numbers and distribution of cellular particulates. Future physiological and biochemical studies with this organism should take these facts into account.

\section{ACKNOWLEDGMENTS}

The authors wish to thank Roger Zieg and Ann Hunt for technical assistance.

\section{LITERATURE CITED}

Cameron, I. L., and E. E. Guile 1965 Nucleolar and biochemical changes during unbalanced growth of Tetrahymena pyriformis. J. Cell. Biol., 26: 845-855.

de Duve, C. 1964 From cytases to lysosomes. Federation Proc., 23; 1045-1049.

Elliott, A. M., and I. J. Bak 1964a The contractile vacuole and related structures in Tetrahymena pyriformis. J. Protozool., 11: 250-261.

- 1964b The fate of mitochondria during aging in Tetrahymena pyriformis. J. Cell. Biol., 20: 113-129.

Elliott, A. M., and J. Hogg 1952 Culture variation in Tetrahymena pyriformis. Physiol. Zool., 25: 318-323.

Erwin, J., and K. Block 1963 Lipid metabolism of ciliated protozoa. J. Biol. Chem., 238: 16181624 .
Hall, R. P. 1953 Protozoology, New York: Prentice-Hall, Inc.

Hermdon, R. M. 1964 Lamellar bodies, an unusual arrangement of the granular endoplasmic reticulum. J. Cell. Biol., 20: 338-342.

Levy, M. R., and O. H. Scherbaum 1965 Gly. coneogenesis in growing and non-growing cultures of Tetrahymena pyriformis. J. Gen. Microbiol., 38: 221-230.

Luft, J. H. 1961 Improvements in epoxy resins embedding methods. J. Biophysic. and Biochem. Cytol., 9; 409.

McCashland, B. W., and J. M. Kronschnabel 1962 Exogenous factors affecting respiration in Tetrahymena pyriformis. J. Protozool, 9: 276-279.

Palade, G. E. 1956 Electron microscopy of mitochondria and other cytoplasmic structures. In Enzymes: Units of biological structure and functions, O. H. Gaebler (editor), New York: Academic Press, Inc., Chapter 9.

Peachey, L. O. 1964 Electron misroscopic observations on the accumulation of divalent cations in intramitochondrial granules. J. Cell. Biol., 20: 95-111.

Rudzinska, M. 1962 The use of a protozoan for studies on aging III. Similarities between young overfed and old normally fed Tokophrya infusionum. A light and electron microscope study. Gerontologia., 6: 206-226.

Sonneborn, T. M. 1957 Breeding systems, reproductive methods, and special problems in protozoa. In: The Species Problem, Washington D.C.: A.A.A.S. pages 155-324.

Steiner, J. W., K. Miyai and M. J. Phillips 1964 Electron microscopy of membrane-particle arrays in liver cells of ethionine-intoxicated rats. Am. J. Path., 44: 169-213.

PLATE 1

EXPLANATION OF FIGURES

Except where indicated the ciliates are fixed in $\mathrm{OsO}_{4}$. Figures 1, 2, and 8 are of young ( 36 hour incubation) cells, all others are seven days or older.

1 A low power micrograph of a whole ciliate grown in a quiet flask for 36 hours. The mitochondria $(M)$ are peripherally located and numerous primary lysosomes (LY) appear in the cytoplasm. The nucleoli (NU) lie near the membrane of the macronucleus (MA). Ciliates under the four culture conditions have essentially the same structure during logarithmic growth. $\times 3,000$.

2 This micrograph is of a cell grown under the same conditions as in figure 1. The moderately dense vacuoles with a unit membrane are probably primary lysosomes (LY). The rough endoplasmic reticulum ( $R E R$ ) often occurs in lamellae which usually lie near a mitochondrion, shown in a grazed section. Dispersed ribosomes are abundant. $\times 27,000$. 

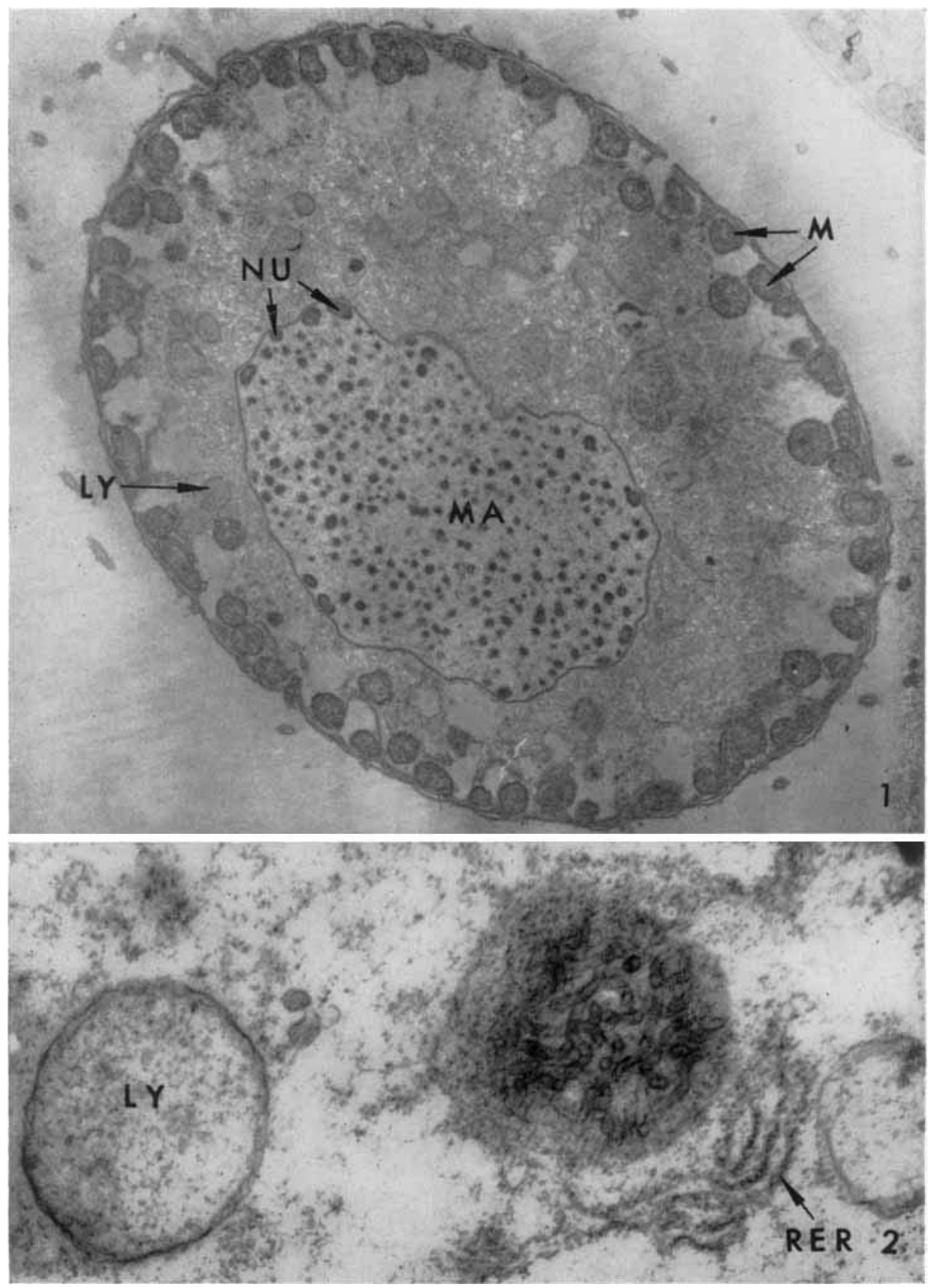
PLATE 2

EXPLANATION OF FIGURE

3 A glutaraldehyde-fixed whole cell, seven days old, cultured in a quiet flask. Note the numerous randomly distributed mitochondria. Lipid droplets $(L)$ are concentrated anterior to the nucleus. An occasional primary lysosome (LY) can be seen. Whereas autophagic vacuoles occur at this stage, none appear in this section. The nucleoli (NU) are large and few in number. $\times 3,000$. 


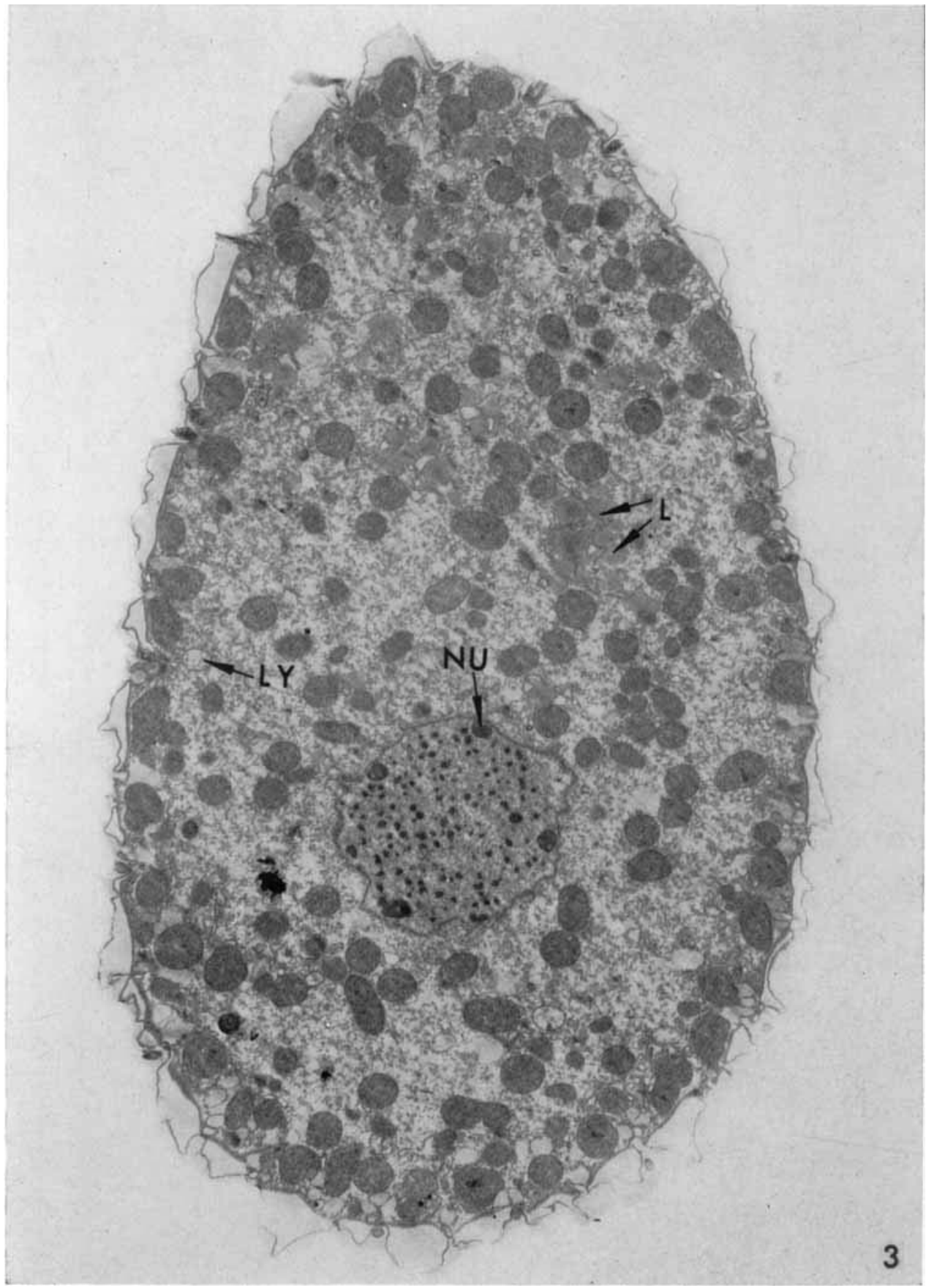


PLATE 3

\section{EXPLANATION OF FIGURES}

4 This cell was grown in a quiet, aerated flask. The mitochondria and lipid droplets are more numerous than in the unaerated flask (fig. 3 ). The lipid is also concentrated anterior to the macronucleus. More of the mitochondria contain dense granules. The nucleoli are small and resemble those of younger cells. The white flecks are due to poor embedding. $\times 2,800$.

5 An enlargement of a cell treated similarly to the one in figure 4 showing RER and stellate lipid droplets $(L) . \times 18,000$. 


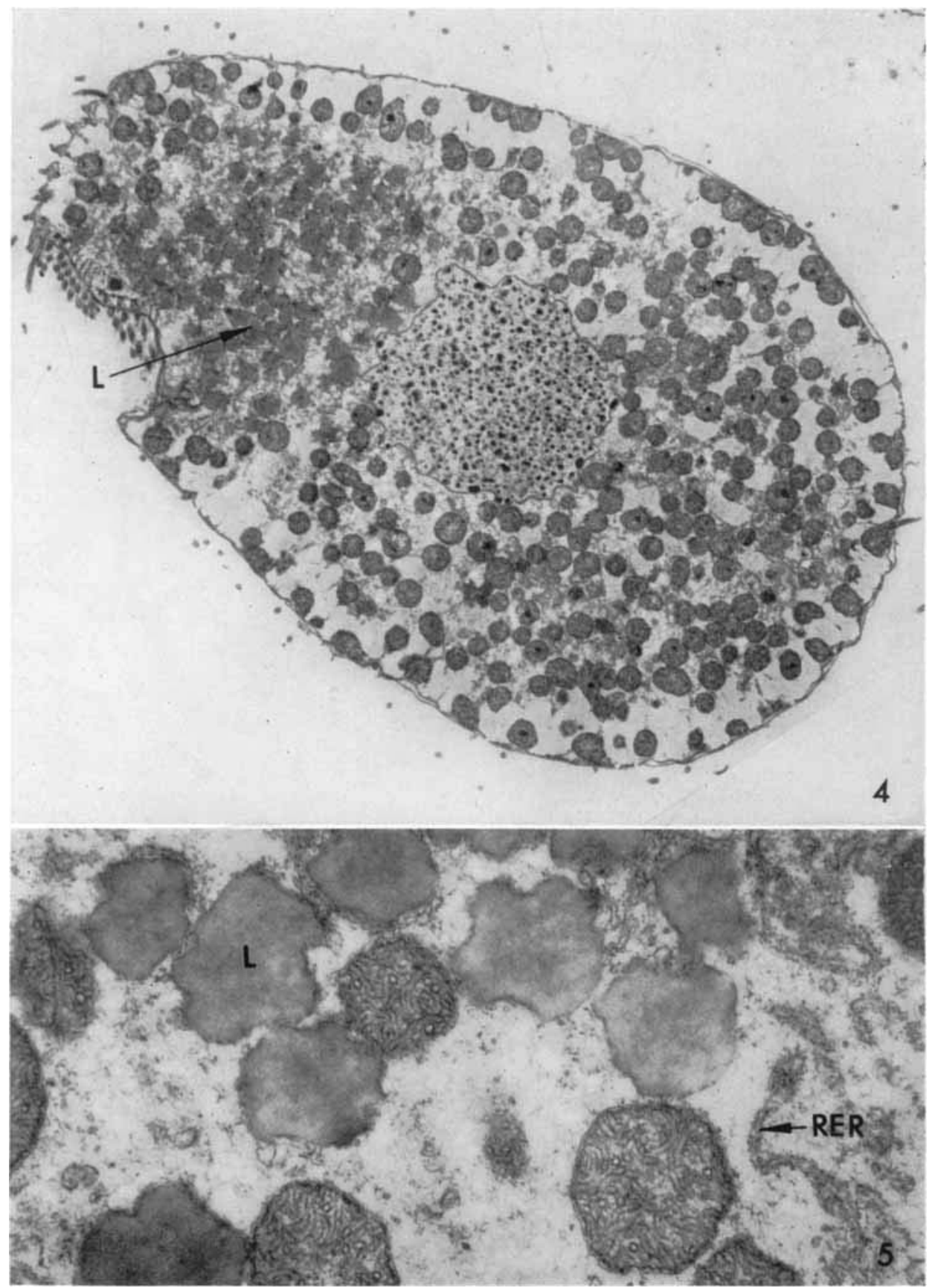


PLATE 4

EXPLANATION OF FIGURE

6 This gluteraldehyde-fixed ciliate was grown in a flask that was stirred but not aerated. Note the peripherally aligned mitochondria $(M)$. Primary lysosomes ( $L Y$ ) and autophagic vacuoles ( $A V$ ) containing a single mitochondrion, appear at random. Food vacuoles (FV) with associated RER occur in this section. Note the absence of lipid droplets. The two large masses of nucleolar material (NU) may represent a stage in fusion of smaller nucleoli. $\times 2,500$. 


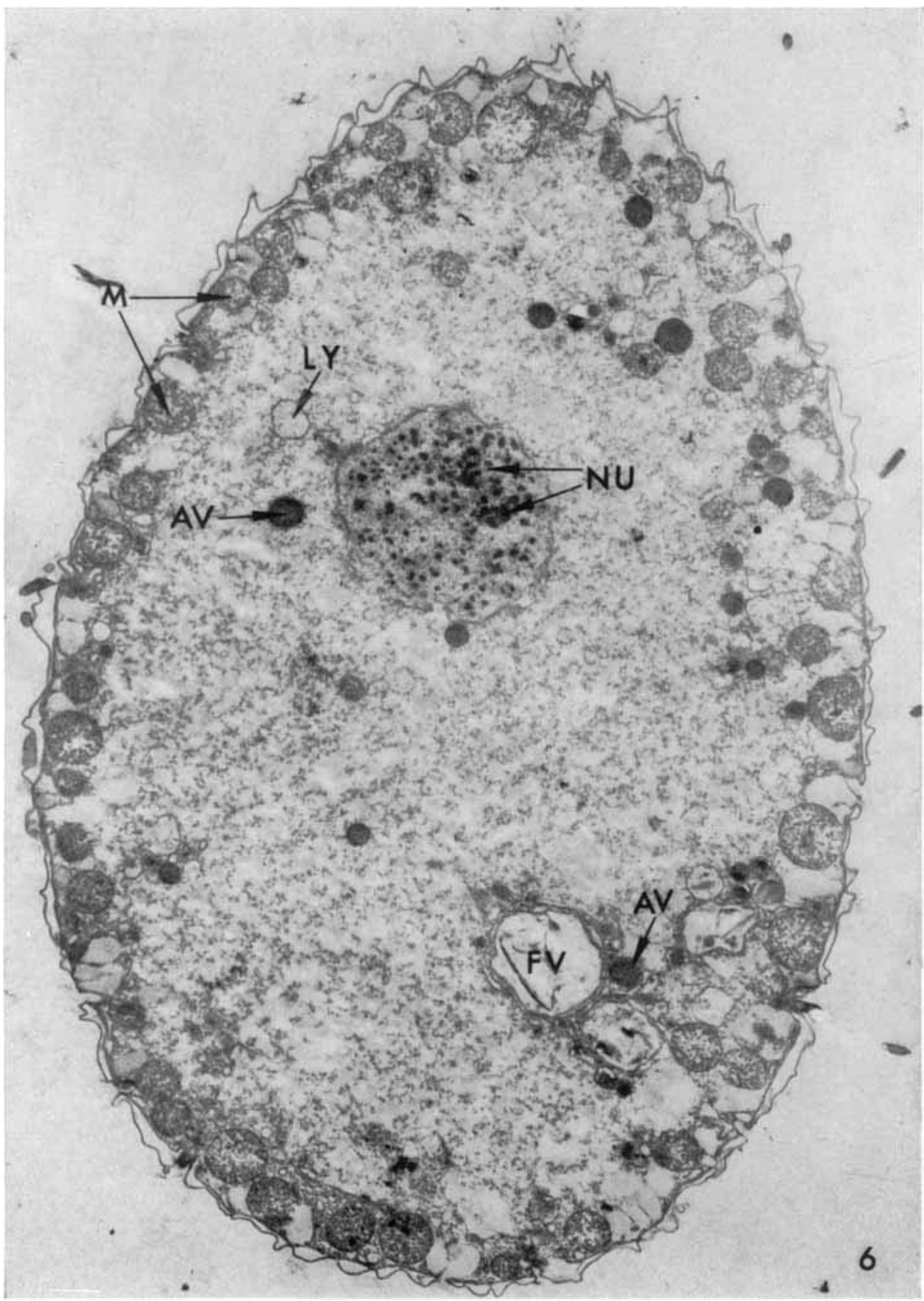


PLATE 5

EXPLANATION OF FIGURES

7 Autophagic vacuoles appear in stationary growth phase ciliates. These may contain one or more mitochondria as shown in this micrograph. These encapsulated mitochondria usually contain a dense granule and the tubules are compacted prior to hydrolysis. $\times 27,000$.

8 This macronucleus is characteristic of logarithmic growth cells. The crescent-shaped nucleoli (NU) are numerous, small, and located on the inner surface of the macronuclear membrane. $\times 6,000$.

9 The nucleoli ( $N U$ ) of stationary growth phase cells are large and often irregularly shaped as shown in this micrograph. They may be located at the periphery of the macronucleus or within the nucleoplasm (see fig. 6). This section is taken to one side of the center of the macronucleus. $\times 10,000$. 

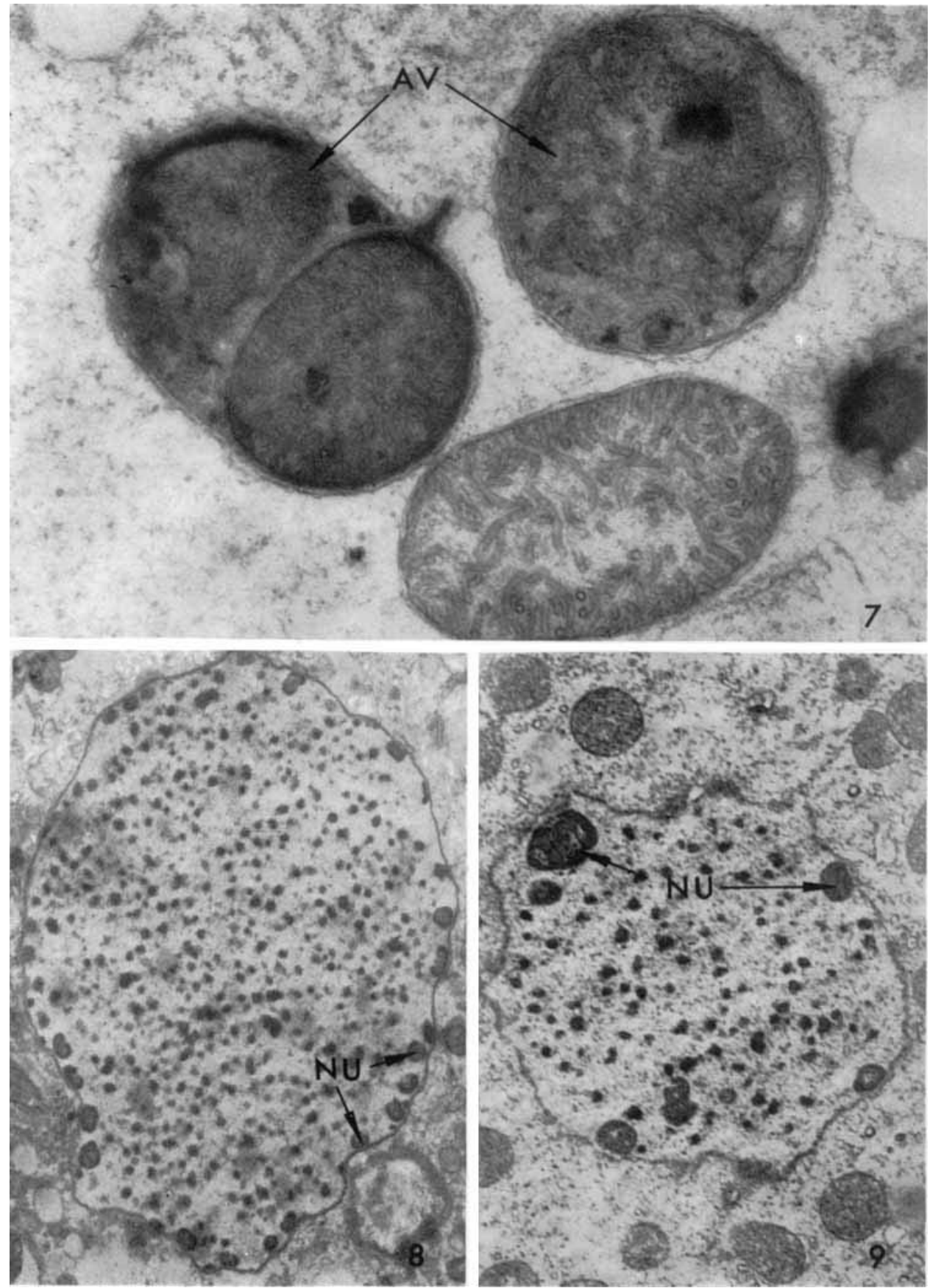


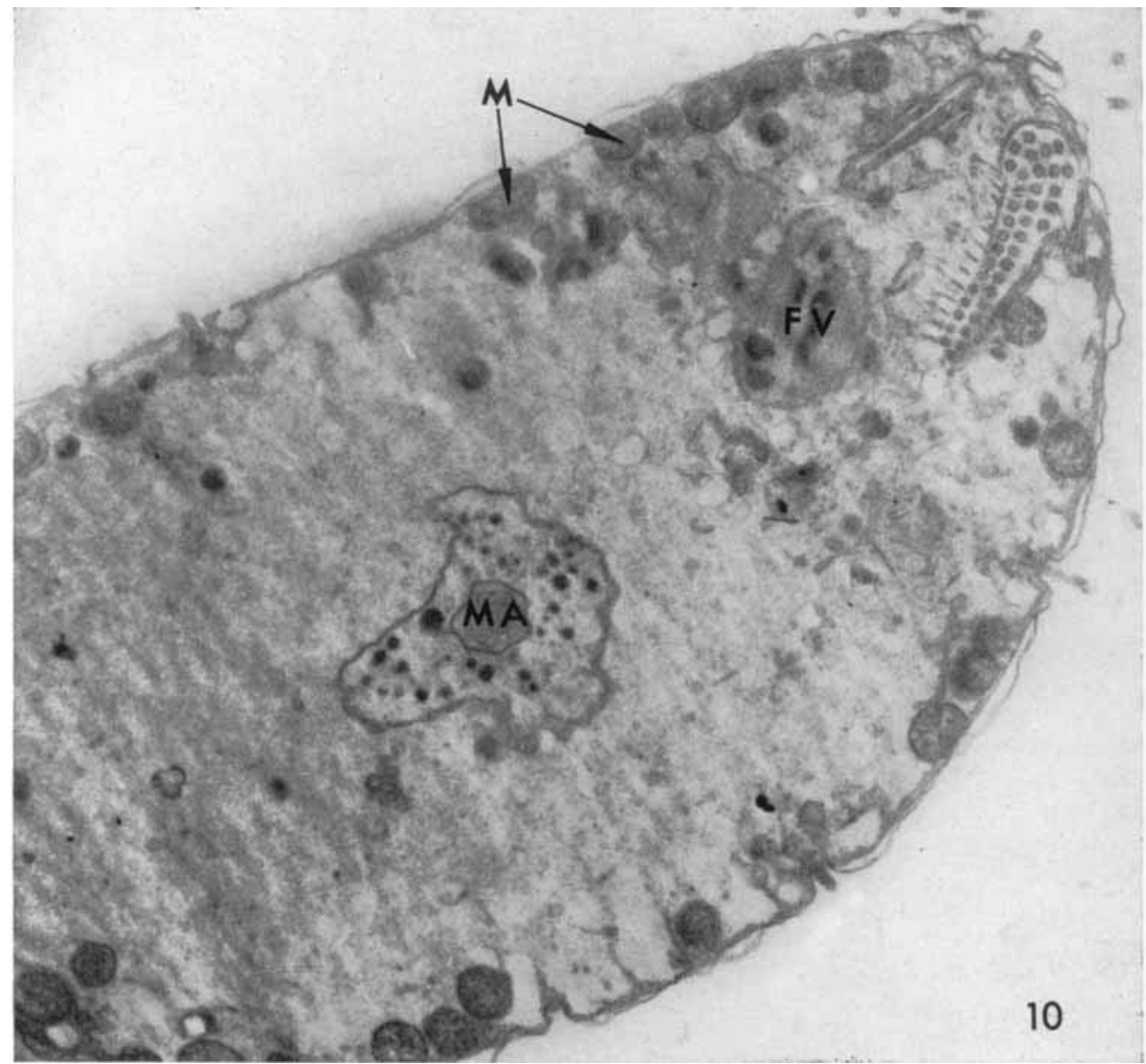

EXPLANATION OF FIGURE

10 This cell was taken from a culture aerated and stirred for 12 days. Note the reduction in numbers of mitochondria $(M)$. Their distribution is peripheral as in young cells (compare with fig. 1). An occasional food vacuole (FV) appears in these sections. The small portion of the macronucleus (MA) shown in this section does not represent a true interpretation of nucleoli in old ciliates. $\times 2,500$. 